\title{
Correction to: Communication about Hereditary Cancers on Social Media: a Content Analysis of Tweets about Hereditary Breast and Ovarian Cancer and Lynch Syndrome
}

\author{
Caitlin G. Allen ${ }^{1} \cdot$ Megan Roberts ${ }^{2} \cdot$ Brittany Andersen $^{3} \cdot$ Muin J. Khoury $^{4}$ \\ Published online: 15 May 2020 \\ (C) American Association for Cancer Education 2020
}

\section{Correction to: Journal of Cancer Education https://doi.org/10.1007/s13187-018-1451-4}

The original version of this article unfortunately contained mistakes.

- Fourth sentence of the Abstract should read:

A total of 47,396 tweets were included in our dataset. The majority were retweets $(60.8 \%)$ and users came from 654 different cities. Most tweets were considered "informational" $(57.5 \%)$ and were designed to provide resources to the public. Individual users $(40 \%)$ were the most common contributors.

- Methods section, last sentence of 1st paragraph should be:

The online version of the original article can be found at https://doi.org/ 10.1007/s13187-018-1451-4

Caitlin G. Allen

calle27@emory.edu

Megan Roberts

megan.roberts@nih.gov

Brittany Andersen

bla@bu.edu

Muin J. Khoury

muk1@cdc.gov

1 Rollins School of Public Health, Emory University, 1518 Clifton Rd, Atlanta, GA 30322, USA

2 The National Cancer Institute, 9609 Medical Center Drive, Rockville, MD 20850, USA

3 Boston University, 704 Commonwealth Avenue, Boston, MA 02215, USA

4 Office of Public Health Genomics, Centers for Disease Control and Prevention, 1600 Clifton Road, Atlanta, GA 30329, USA
A total of 63,770 tweets were originally identified using our search strategy. The final sample consisted of 47,396 tweets.

- Methods section, a new paragraph should be inserted after the 4th paragraph:

After coding was complete, we then cleaned the dataset to remove tweets that were misclassified. We discovered that the hashtag BRCA was also used during this time period to reference the Better Care Reconciliation Act (BCRA). Thus, we removed tweets that were referencing the BCRA but used the hashtag of BRCA. This resulted in a final sample of 47,397.

- Results section, 1st paragraph should be:

Our final sample included 47,397 tweets that included a hashtag related to HBOC or LS. Users represented 654 cities and 105 countries. The majority of tweets were retweets of previous conversations; just over one-third of tweets were original content $(35.5 \%)$.

- Results section, first two sentences of the 2nd paragraph should read:

Categories and emotions of tweets were most frequently "neutral" (73.4\%). Most tweets were classified as informational (57.5\%), other types of tweets included opinion (18.6\%) and testimonial (11.8\%). Types of information included real world applications (48.4\%), practice/implementation (23.6\%), and policy $(11.1 \%)$ (Tables 1 and 2).

- Results section, 1st sentence of the 3rd paragraph should read:

The most frequent contributors to the Twitter conversation included the "Anti-Cancer Club" (@anticancerclub) and a combination of individual users (Table 3). 
- Discussion section, last five sentences of the 4th paragraph should read:

Finally, we identified tweets that used the hashtag BRCA in reference to the Better Care Reconciliation Act (BCRA) after completing coding using machine learning. We removed these tweets; however, we were unable to re-classify the new dataset using machine learning techniques. The volume of unrelated BRCA tweets could have impacted machine learning or have led to a slightly skewed machine categorization. Our team worked to reduce this impact by removing irrelevant tweets post-machine learning and evaluating the machine's coding of other categories after identification of the incorrect acronym usage. Future studies can work toward identification of as many irrelevant tweets from acronym usage or misspelling at the data collection stage to best avoid this same issue.

- $\quad$ Presented here also are the corrected Tables 1, 2 and 3.
Table 1 Descriptive Information about Tweets and Users

\begin{tabular}{|c|c|c|}
\hline Category & $\mathbf{N}$ & $\%$ \\
\hline \multicolumn{3}{|l|}{ Post Type } \\
\hline Reply & 1740 & 3.7 \\
\hline Retweet & 28,834 & 60.8 \\
\hline Tweet & 16,822 & 35.5 \\
\hline \multicolumn{3}{|l|}{ Type of Tweet } \\
\hline Informational & 27,259 & 57.5 \\
\hline Testimonial & 5597 & 11.8 \\
\hline Opinion & 8827 & 18.6 \\
\hline Research & 1496 & 3.2 \\
\hline Other & 2041 & 4.3 \\
\hline \multicolumn{3}{|l|}{ Type of Information } \\
\hline New discoveries in the field & 4601 & 9.7 \\
\hline Practice/implementation & 11,169 & 23.6 \\
\hline Real world applications & 22,927 & 48.4 \\
\hline Policy & 5259 & 11.1 \\
\hline Other & 1101 & 2.3 \\
\hline Number of cities/states & 654 & \\
\hline Number of Countries & 105 & \\
\hline \multicolumn{3}{|l|}{ Categories } \\
\hline Negative & 3104 & 6.6 \\
\hline Neutral & 34,847 & 73.4 \\
\hline Unclassified & 1726 & 3.64 \\
\hline Positive & 7719 & 16.3 \\
\hline \multicolumn{3}{|l|}{ Emotion } \\
\hline Anger & 553 & 1.2 \\
\hline Disgust & 622 & 1.3 \\
\hline Fear & 1370 & 2.9 \\
\hline Joy & 8864 & 18.7 \\
\hline Neutral & 29,609 & 62.5 \\
\hline Sadness & 1538 & 3.2 \\
\hline Surprise & 23 & 0.1 \\
\hline Unclassified & 4817 & 10.2 \\
\hline Followers & 5217 & $0-3,741,121$ \\
\hline Following & 2849 & $0-476,597$ \\
\hline \multicolumn{3}{|l|}{ Gender } \\
\hline Female & 16,496 & 63.2 \\
\hline Male & 9623 & 36.8 \\
\hline
\end{tabular}


Table 2 Examples of Tweets and Information

\section{Type of Tweet}

Informational

Testimonial

Opinion

Research

Other

Type of Information

New discoveries in the field

Practice/implementation

Real world applications

Policy

Other
Do you have a genetic mutation? Visit our website for lots of information and advice $>$ https://tco/vzHHLS9X6q \#BRCA \#lynch https://t.co/aPAjuOs0Dy

"\#Mammograms are considered to be life-saving for \#women in their \#thirties with \#BRCA mutations. @ BrynaSF edu https://t.co/hz6MflbK89

"How do we know \#BRCA genes lost their function?" Explained by our CTO, Zhenyu Xu @EPFL Applied \#MachineLearning Days @appliedmldays https://t.co/JCwQKXpFeE

How do I tell my \#child that I have \#cancer? https://t.co/htZN6ug2jh \#coloncancer \#Lynchsyndrome \#prostatecancer https://t. co/HUFgcMc6O1

On her deathbed, mom asks daughter to promise to have life-saving surgery https://t.co/tSu9JO5eTj via @ TODAYshow \#brca RT @ anticancerclub \#Cancer and a crisis of confidence https://t.co/gRrhRL27JY \#LynchSyndrome

Re-learning what works for your body during and after treatment https://t.co/Myg5z2N8hc \#LynchSyndrome \#Cancer \#UterineCancer

Practicing \#kindness and relaxing beliefs to \#heal https://t.co/Myg5z34JFM \#LynchSyndrome \#Cancer

RT @ Ashworth SF Just out, our review of the state of the art: PARP inhibitors \#BRCA @UCSFCancer @UCSF BRCA https://t.co/SGQbtg2UHt https://t.co/J5YZUi4tfx

\#Prophylactic \#Treatment Reduces Neratinib-Related Toxicity in \#HER2+ \#BreastCancer https://t.co/omn6raCYcm via @ onclive \#brca \#cancer

How should we screen \#endometrial cancer for \#Lynchsyndrome? Manchester Mtg @HHampel1 @ karenluMD @TMSnowsill @CaPP3 https://t.co/8YIL3RCrpl

RT @SFBusinessTimes This week’s \#biotech \#podcast: Beyond @ AngeelinaJolie: Tracking down the cancer-causing \#BRCA gene mutation https://t.co/dy3gyqWYWe https://t.co/S4SR4dJ0mI

RT @ Roche Are you aware of the risk factors of ovarian cancer? https://t.co/bDBZHwR7yX \#BRCA \#HereditaryCancer https://t.co/DrrsGJAbKo

RT @AmbryGenetics Genetic testing is recommended if you have close relatives w a known \#BRCA mutation. https:/t. co/9BK5S8tTuf \#BreastCancerAwarenessMonth https://t.co/7tDUTnWQPA

RT @SCSanderson Should everyone be offered BRCA genetic testing for cancer risk, regardless of their personal or family history? \#BRCA \#cancer

RT @ sloan_kettering “If I have \#BRCA1 or \#BRCA2 gene mutation should I get a preemptive \#mastectomy?" Dr. Barrio weighs in https://t.co/UAdGHimDfM \#BCSM \#BCAM https://t.co/45DB582O3e

Adapting to body changes during \#cancer treatment https://t.co/fnaXDiS2LU \#LynchSyndrome https://t.co/cbubC70fa2

Helping people and raising awareness @ catnd https://t.co/Myg5z34JFM \#LynchSyndrome

March is \#ColonCancerAwarenessMonth! \#LynchSyndrome https://t.co/G5eeSGbyN3

RT @ Rewire_News How uncertainty around the new health-care law could hurt people at risk for breast cancer https://t. co/p2UIFA4dve \#AHCA \#BRCA https://t.co/h2zG8C4EVV

RT @ ShannonWestin Another great option for \#BRCA mutant recurrent \#ovariancancer @US_FDA approves Lynparza \#PARPi maintenance https://t.co/Mrxkf8rJit \#gyncsm

How do I tell my \#child that I have \#cancer? https://t.co/htZN6ug2jh \#coloncancer \#Lynchsyndrome \#prostatecancer https:/t. co/HUFgcMc6O1 
Table 3 Most Common Tweeters

\begin{tabular}{|c|c|c|c|c|}
\hline Account & Name & $\begin{array}{l}\text { Number of } \\
\text { Tweets }\end{array}$ & Account Information & Type of Account \\
\hline @ anticancerclub & Anti-Cancer Club & 2261 & $\begin{array}{l}\text { On a mission to help people craft a personalized } \\
\text { anti-cancer life. Information, education and a } \\
\text { chance to connect! }\end{array}$ & Organization \\
\hline @ BRCAresponder & Amy Byer Shainman & 1354 & $\begin{array}{l}\text { Education-Advocacy-Support for \#BRCA and } \\
\text { other \#hereditarycancer syndromes. Exec. } \\
\text { Producer @ pinkandbluedocs Co-creator } \\
\text { @Genc_SM \#GenCSM }\end{array}$ & Individual User \\
\hline @ LguzzardiM & Lisa M Guzzardi, RN & 976 & $\begin{array}{l}\text { \#PatientAdvocate dedicated to providing up to } \\
\text { date research for } 2.9 \mathrm{~K} \text { consumers at risk \& } \\
\text { clinicians \#JournalClub \#bcsm \#gyncsm } \\
\text { \#PancChat \#BRCA \#hereditarycancer }\end{array}$ & Provider \\
\hline @ BRCAUmbrella & BRCA Umbrella & 799 & $\begin{array}{l}\text { Join others sharing experiences of living with a } \\
\text { BRCA mutation or a high cancer risk at } \\
\text { http://www.brcaumbrella.ning.com }\end{array}$ & Organization \\
\hline @ karenBRCAMTL & Karen Lazarovitz & 797 & $\begin{array}{l}\text { Creator \#BRCA Sisterhood \& Supportgroup } \\
\text { \#Montreal \#breastcancer\#hereditarycancer } \\
\text { \#genetictesting \#publicspeaker my story } \\
\text { \#mastectomy \#tattoos link below }\end{array}$ & Individual User \\
\hline @ BRCAinfo & Tobey & 745 & $\begin{array}{l}\text { \#BRCA \#ConsumerAdvocate \#research } \\
\text { \#immunotherapy \#BladderCancer \#patient } \\
\text { \#family \#health \#dogs \#coffee \#books }\end{array}$ & Individual User \\
\hline @GenC_SM & \#GenCSM & 561 & $\begin{array}{l}\text { \#Tweetchat \& community for those interested in } \\
\text { \#Genetics and \#HereditaryCancer syndromes. } \\
\text { Co-creators: @ MyGeneCounsel } \\
\text { \#BRCAresponder@_GeorgiaHurst }\end{array}$ & Online Community \\
\hline @CancerRoadTrip & \#CancerRoadTrip & 511 & $\begin{array}{l}\text { Inspiration, joy and discovery through travel. Life } \\
\text { is an adventure. Live it! Award Winning } \\
\text { \#TravelBlogger, Aspiring Photographer, } \\
\text { Instagram: @CancerRoadTrip }\end{array}$ & Individual User \\
\hline @ BreastAdvocate & Breast Advocate & 446 & $\begin{array}{l}\text { Free \#BreastCancer } \\
\quad \text { \#Surgery\#SharedDecisionMaking App for } \\
\text { newly-diagnosed, Survivors, Thrivers \& } \\
\text { Previvors. Co-created by breast cancer special- } \\
\text { ists \& patient advocates. }\end{array}$ & Organization \\
\hline @MNWilliams74 & Michelle Williams & 559 & No longer active & Individual User \\
\hline @brcafndn & BRCA Foundation & 394 & No longer active & Organization \\
\hline @NickiDurlester & Nicki B. Durlester & 350 & $\begin{array}{l}\text { \#BRCA2 \#BreastCancer \#Survivor, \#Author \& } \\
\text { Founder \#BeyondthePinkMoon support group } \\
\text { @ facebook for survivors, previvors, MDs \& } \\
\text { HCPs w/ } 7.8 \mathrm{k} \text { members. \#bcsm \#gyncsm }\end{array}$ & Online Community \\
\hline @Linktheribbons & Link The Ribbons & 340 & $\begin{array}{l}\text { Raising Cancer awareness across the spectrum. } \\
\text { Collaboration and cooperation by sharing } \\
\text { information, experience and hope. United we } \\
\text { are stronger. \#Linktheribbons }\end{array}$ & Online Community \\
\hline @ drkdhoffman & Kathleen D. Hoffman & 324 & $\begin{array}{l}\text { Healthcare Consumer Engagement, Healthcare } \\
\text { Content Writer, SocialMedia, Blogging, } \\
\text { Marketing, Health Communication, Health } \\
\text { Literacy, Social Science Researcher }\end{array}$ & Researcher \\
\hline @ carefreemajorca & Hamill Care & 310 & $\begin{array}{l}\text { \#accessible \#holidays, \#tourism and } \\
\text { \#DisabledSport in \#Mallorca for those with } \\
\text { \#disability and \#seniors. 24/7 \#CARE }\end{array}$ & Individual User \\
\hline @ DiepFlapBreast & PRMA Plastic Surgery & 272 & $\begin{array}{l}\text { PRIMA \#PlasticSurgery specializes in advanced } \\
\text { \#breastreconstruction after \#breastcancer - } \\
\text { DIEP, SIEA, GAP, TUG, Fat Grafting \& } \\
\text { One-Step. }\end{array}$ & Provider Organization \\
\hline @luisa_tasayco & Maria & 256 & $\begin{array}{l}\text { PhD Chemistry Emerita Professor } \\
\text { Chemistry/Biochemistry CCNY } \\
\text { Biochemistry/Biophysics Graduate Center } \\
\text { CUNY }\end{array}$ & Individual User \\
\hline @MBCC_MHBT & Male Breast Cancer & 247 & $\begin{array}{l}\text { The Male Breast Cancer Coalition (MBCC) is } \\
\text { your resource for MEN with breast cancer. Our } \\
\text { reach is global and continues to grow daily. } \\
\text { \#MenHaveBreastsToo }\end{array}$ & Organization \\
\hline @ IrinaBrooke1 & Mutant Strong & 224 & & Individual User \\
\hline
\end{tabular}


Table 3 (continued)

\begin{tabular}{|c|c|c|c|c|}
\hline Account & Name & $\begin{array}{l}\text { Number of } \\
\text { Tweets }\end{array}$ & Account Information & Type of Account \\
\hline @ drchrysopoulo & PRMA Plastic Surgery & 215 & $\begin{array}{l}\text { BRCA2 BC survivor Creator of Mutant Strong on } \\
\text { Facebook. Mutant_Strong on Instagram. } \\
\text { Patient, BRCA/HBOC advocate. Blog: } \\
\text { mutantstrong.com } \\
\text { \#Surgeon \& President at PRMA \#PlasticSurgery. } \\
\text { Tweet a lot about \#BreastReconstruction \& } \\
\text { \#SharedDecisionMaking. Creator of } \\
\text { @BreastAdvocate App \& @ InvCibleScars }\end{array}$ & Provider \\
\hline
\end{tabular}

Publisher's Note Springer Nature remains neutral with regard to jurisdictional claims in published maps and institutional affiliations. 